\title{
Living donor liver transplantation for unresectable colorectal liver metastasis: Report of a case with 13-year follow-up without recurrence
}

\author{
Jin Uk Choi, Shin Hwang, Dong-Hwan Jung, Gil-Chun Park, Chul-Soo Ahn, \\ Ki-Hun Kim, Deok-Bog Moon, Tae-Yong Ha, Gi-Won Song, and Sung-Gyu Lee

\begin{abstract}
Division of Hepatobiliary Surgery and Liver Transplantation, Department of Surgery, Asan Medical Center, University of Ulsan College of Medicine, Seoul, Korea
\end{abstract}

\begin{abstract}
The feasibility of liver transplantation (LT) for colorectal liver metastasis (CRLM) is still under investigation with only a limited number of LT cases in literature. CRLM is the most common type of liver metastasis, but it was considered as a contraindication to LT for a long time because of poor outcomes. We presented a case of living donor liver transplantation (LDLT) performed in a patient with liver cirrhosis and CRLM. The patient was a 49-year-old female with sigmoid colon cancer and synchronous multiple CRLM. She underwent anterior resection for sigmoid colon cancer and 7 sessions of chemotherapy for CRLM. She suffered from esophageal varix bleeding due to chemotherapy-associated liver cirrhosis. Because of liver cirrhosis and multiple CRLM, the patient underwent LDLT operation using a modified right lobe graft. Serum chorioembryonic antigen level was $220 \mathrm{ng} / \mathrm{mL}$ at LT. Explant liver pathology showed multiple metastatic adenocarcinomas of colonic origin, up to $4.7 \mathrm{~cm}$ in the greatest dimension. The patient did not receive any specific anti-tumor treatment after LT. She is doing well without any tumor recurrence to date for more than 13 years after the LDLT operation. The experience on our case and literature review suggest that CRLM is not always contraindicated for LT because some selected patients showed improved long-term survival outcomes. (Ann Hepatobiliary Pancreat Surg 2020;24:188-191)
\end{abstract}

Key Words: Colorectal cancer; Liver metastasis; Living donor liver transplantation; Adenocarcinoma

\section{INTRODUCTION}

The outcomes of liver transplantation (LT) for malignancy have improved significantly over the years. Besides hepatocellular carcinoma, LT has been used to manage several other types of cancers, such as cholangiocarcinoma, neuroendocrine liver metastases, and more rarely hepatoblastoma and angiosarcoma. ${ }^{1,2}$ The feasibility of LT for colorectal liver metastasis (CRLM) has been explored in small series, however, the interest to develop this field has been rapidly expanding. ${ }^{3}$ LT can be considered as a definitive option for surgical management of liver malignancy since the entire liver organ with all metastatic tumor burden is replaced. CRLM is the most common type of liver metastasis, but it had been considered as a contraindica- tion to LT for a long time because of poor posttransplant outcomes. $^{4}$

We herein present a case of a patient with unresectable multiple CRLM who underwent living donor liver transplantation (LDLT) and is still alive to date for 13 years without any tumor recurrence.

\section{CASE}

The case was a 49-year-old female patient who had sigmoid colon cancer with synchronous liver metastasis and underwent anterior resection at the outside hospital in October 2004 (Fig. 1A). The synchronous multiple liver metastasis was treated with 7 sessions of hepatic-arterial infusion (HAI) chemotherapy. However, this chemotherapy

Received: March 24, 2020; Revised: March 28, 2020; Accepted: March 29, 2020

Corresponding author: Shin Hwang

Department of Surgery, Asan Medical Center, University of Ulsan College of Medicine, 88 Olympic-ro 43-gil, Songpa-gu, Seoul 05505, Korea Tel: +82-2-3010-3930, Fax: +82-2-3010-6701, E-mail: shwang@amc.seoul.kr

Copyright (C) 2020 by The Korean Association of Hepato-Biliary-Pancreatic Surgery

This is an Open Access article distributed under the terms of the Creative Commons Attribution Non-Commercial License (http://creativecommons.org/ censes/by-nc/4.0) which permits unrestricted non-commercial use, distribution, and reproduction in any medium, provided the original work is properly cited. Annals of Hepato-Biliary-Pancreatic Surgery - pISSN: 2508-5778 - elSSN: 2508-5859 

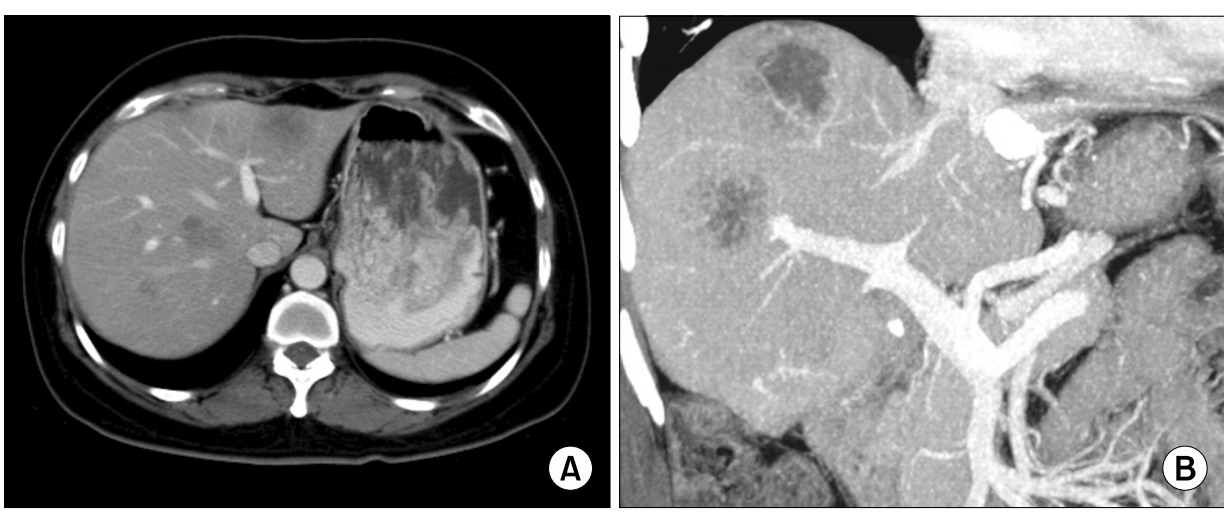

Fig. 1. Pretransplant computed tomography (CT) findings. (A) A CT image taken at the time of colon cancer surgery (October 2004) showed multiple liver metastasis from sigmoid colon cancer. (B) A CT image taken at liver transplantation (January 2007) showed multiple unresectable colorectal liver metastasis.

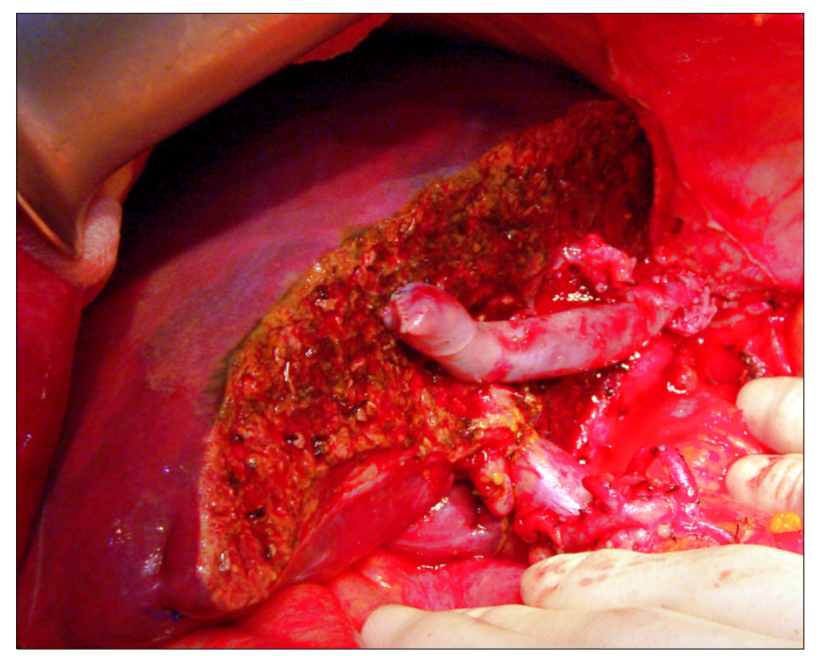

Fig. 2. Intraoperative photograph of the liver graft implantation. There was a small area of hepatic venous congestion after reperfusion with portal blood flow.

showed only partial responses. The patient also suffered from esophageal varix bleeding due to liver cirrhosis that might be associated with the repeated sessions of HAI chemotherapy (Fig. 1B). She did not show any evidence of hepatitis $\mathrm{B}$ or $\mathrm{C}$ infection.

The patient was transferred to our institution to evaluate for LDLT. Although CRLM was generally regarded as a contraindication of LT at that time, a LDLT operation was planned as a definitive treatment for liver cirrhosis and as a potential surgical treatment for CRLM. The model for end-stage liver disease score was 8 . The pretransplant work-up revealed that CRLM was liver-limited and the colonic operation site was disease-free. Serum chorioembryonic antigen (CEA) level was $220 \mathrm{ng} / \mathrm{mL}$ at the time of LT.

In January 2007, a LDLT operation using a modified right lobe graft was performed. The donor was the 46-

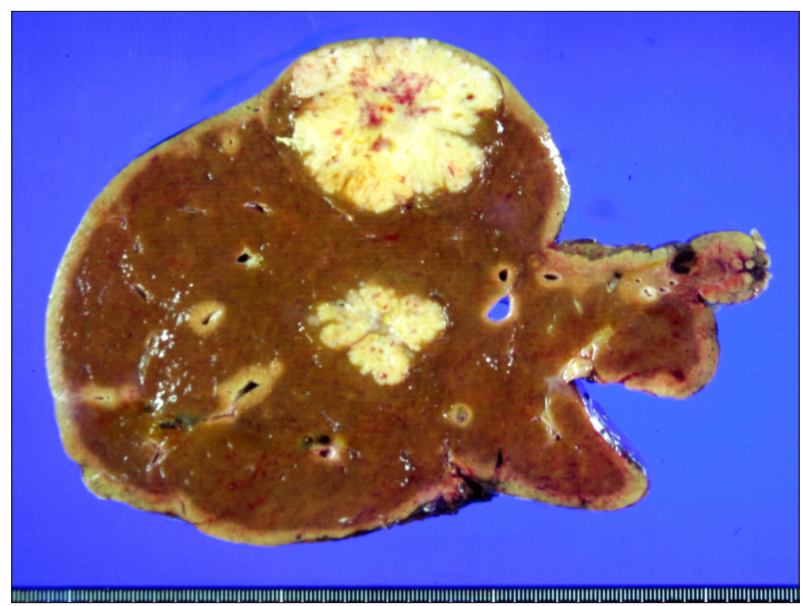

Fig. 3. Gross photograph of the explant liver. There were multiple metastatic adenocarcinomas of colonic origin combined with liver cirrhosis.

year-old patient's sister. The right liver graft weighed 540 $\mathrm{g}$ and the graft-recipient weight ratio was 0.88 . Standardized surgical procedures of LDLT were used for implantation of the modified right lobe graft (Fig. 2). The graft middle hepatic vein was reconstructed with a cryopreserved iliac vein homograft. The graft right hepatic vein and middle hepatic vein conduits were separately anastomosed with the recipient inferior vena cava. The portal vein and hepatic artery were reconstructed as endto-end anastomosis. There were two right hepatic duct openings, which were reconstructed with double hepaticojejunostomy because the recipient common bile duct was resected to enhance the surgical curability. The explant liver pathology showed multiple metastatic adenocarcinomas of colonic origin, up to $4.7 \mathrm{~cm}$ in the greatest dimension (Fig. 3).

Posttransplant recovery of the graft liver function was uneventful, but the abdominal drain turned to be bloody 
at day 7. There was an active bleeding from the right inferior phrenic artery, which might have been injured during the LDLT operation. This bleeding was controlled with percutaneous embolization of the right inferior phrenic artery. Thereafter, there was no event requiring any specific treatment (Fig. 4A). The reconstructed middle hepatic vein conduit was shrunken during the follow-up imaging studies (Fig. 4B).

After LDLT, the patient did not receive any specific anti-tumor treatment for sigmoid colon cancer liver metastasis. The maintenance immunosuppressant was tacrolimus only. The patient is doing well without any tumor recurrence for more than 13 years after LT.

\section{DISCUSSION}

The first experiences of LT for CRLM failed to demonstrate any survival benefit, which led to the procedure being abandoned for CRLM. Mühlbacher et al. ${ }^{4}$ presented in 1991 the largest series consisting of 25 patients transplanted for CRLM, reporting a dismal 5-year overall survival of $12 \%$. With advances in the fields of LT and surgical oncology in addition to a relatively large pool of donor grafts available in Norway, a randomized controlled trial on LT for CRLM (SEcondary CAncer [SECA] study) was initiated. In 2013, Hagness et al. ${ }^{5}$ reported the results of the SECA study where a total of 21 patients were transplanted for CRLM, in which the 5-year patient survival was $60 \%$ and the 1 -year disease-free survival was $35 \%$ with a median of 27 months follow-up. Toso et al. ${ }^{6}$ presented the results of a multi-center retrospective cohort study of 12 patients who underwent LT for CRLM. Most of their patients had synchronous diseases and almost all patients received preoperative chemotherapy. They reported 1-year and 5-year survival rates of $83 \%$ and $50 \%$, and 1 -year and 5 -year disease-free survival rates of $56 \%$ and $38 \%$, respectively.

Patients with CRLM are being treated with several consecutive treatment modalities, but the majority will ultimately experience disease recurrence. As LT emerges as a potential therapeutic option, we can anticipate the need to evaluate the impact of this treatment strategy compared with other standard multimodal treatments. The data available to date on LT for CRLM reflects a better understanding of disease biology and growing enthusiasm to offer this treatment. However, there still remains skepticism and reluctance to adopt LT based on the fear of wasting valuable resources that would be otherwise available to the LT community as well as futile sacrifice of living donors. $^{2}$

The indication of LT for CRLM is unresectable liver-limited metastasis, but its selection criteria are not defined yet. Dueland et al. ${ }^{7,8}$ observed that four patients with metachronous diseases and tumor-negative nodes in the primary tumor are still alive after 6 to 10 years of LT suggesting that by refining the selection of patients even better long term survival and possible cure in a proportion of cases may be achieved.

One available therapeutic option for unresectable liver-limited CRLM is HAI chemotherapy. LT and HAI for CRLM may share similar indications in terms of anatomically unresectable, liver-limited disease burden with relatively favorable disease biology. ${ }^{2,9-11}$ As the experience with chemotherapy increases, there will be a cohort of patients who suffer from liver cirrhosis that is associated with chemotherapy. If the CRLM is well controlled, such patients with liver cirrhosis would be considered to be LT candidates. In the present patient, repeated sessions of
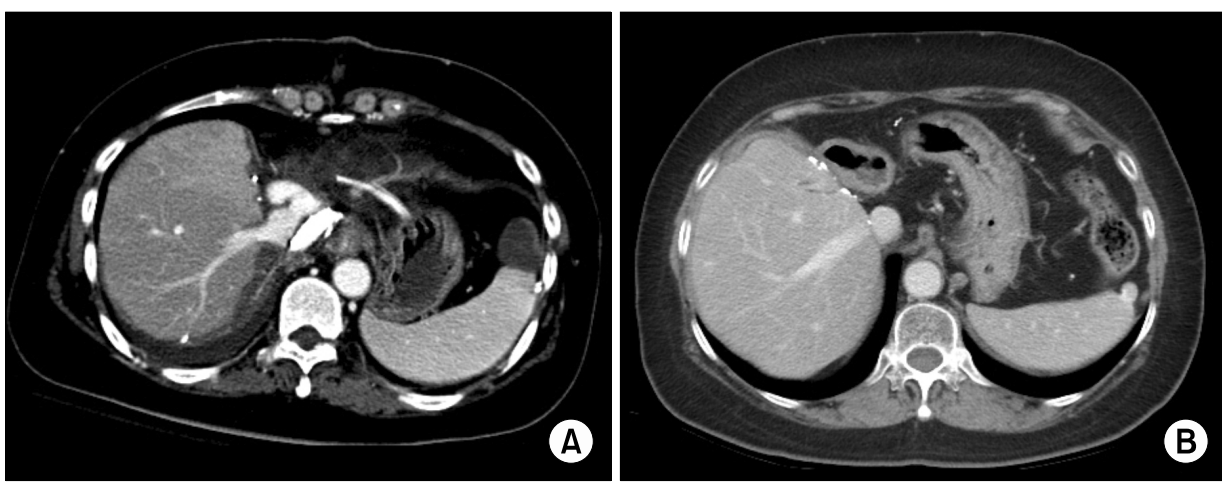

Fig. 4. Posttransplant computed tomography (CT) findings. (A) A CT image taken at 2 weeks after liver transplantation showed no abnormal finding. (B) A CT image taken at 12 years after liver transplantation showed no evidence of tumor recurrence. 
HAI chemotherapy might have induced progression of liver cirrhosis, but CRLM was not well controlled, thus there remained a high risk of posttransplant tumor recurrence.

In literature, most LTs for CRLM were performed by using deceased donor liver grafts, but there was a case report with LDLT in Latin America. ${ }^{12}$ In our institution where more than 5,000 cases of LDLT have been performed, the present case was the only case of LDLT for CRLM because CRLM is still not regarded as an eligible indication of either LDLT or deceased donor LT.

Experience on our case and literature review suggest that CRLM is not always contraindicated for LT because some selected patients showed improved long-term survival outcomes.

\section{CONFLICT OF INTEREST}

No potential financial conflicts or other conflicts of interest exist for any of the authors of this article.

\section{ORCID}

Jin Uk Choi: https://orcid.org/0000-0001-8078-0593

Shin Hwang: https://orcid.org/0000-0002-9045-2531

Dong-Hwan Jung: https://orcid.org/0000-0001-5984-023X

Gil-Chun Park: https://orcid.org/0000-0003-1631-3258

Chul-Soo Ahn: https://orcid.org/0000-0002-3844-3646

Ki-Hun Kim: https://orcid.org/0000-0002-4016-0995

Deok-Bog Moon: https://orcid.org/0000-0002-8209-3540

Tae-Yong Ha: https://orcid.org/0000-0001-9932-0212

Gi-Won Song: https://orcid.org/0000-0002-4235-0434

Sung-Gyu Lee: https://orcid.org/0000-0001-9161-3491

\section{AUTHOR CONTRIBUTIONS}

Conceptualization: SH. Data curation: SH, DHJ. Formal analysis: SH, GCP. Methodology: CSA, KHK, DBM, TYH, GWS. Project administration: SH, SGL. Visualiza- tion: SH. Writing - original draft: SH, JUC. Writing - review \& editing: $\mathrm{SH}$.

\section{REFERENCES}

1. Hoti E, Adam R. Liver transplantation for primary and metastatic liver cancers. Transpl Int 2008;21:1107-1117.

2. Simoneau E, D'Angelica M, Halazun KJ. Liver transplantation for colorectal liver metastasis. Curr Opin Organ Transplant 2019;24:175-181.

3. Gorgen A, Muaddi H, Zhang W, McGilvray I, Gallinger S, Sapisochin G. The new era of transplant oncology: liver transplantation for nonresectable colorectal cancer liver metastases. Can J Gastroenterol Hepatol 2018;2018:9531925.

4. Mühlbacher F, Huk I, Steininger R, Gnant M, Götzinger P, Wamser $\mathrm{P}$, et al. Is orthotopic liver transplantation a feasible treatment for secondary cancer of the liver? Transplant Proc 1991;23(1 Pt 2):1567-1568.

5. Hagness M, Foss A, Egge TS, Dueland S. Patterns of recurrence after liver transplantation for nonresectable liver metastases from colorectal cancer. Ann Surg Oncol 2014;21:1323-1329.

6. Toso C, Pinto Marques H, Andres A, Castro Sousa F, Adam R, Kalil A, et al.; Compagnons Hépato-Biliaires Group. Liver transplantation for colorectal liver metastasis: survival without recurrence can be achieved. Liver Transpl 2017;23:1073-1076.

7. Dueland S, Guren TK, Hagness M, Glimelius B, Line PD, Pfeiffer P, et al. Chemotherapy or liver transplantation for nonresectable liver metastases from colorectal cancer? Ann Surg 2015;261:956-960.

8. Dueland S, Foss A, Solheim JM, Hagness M, Line PD. Survival following liver transplantation for liver-only colorectal metastases compared with hepatocellular carcinoma. Br J Surg 2018; 105:736-742.

9. D’Angelica MI, Correa-Gallego C, Paty PB, Cercek A, Gewirtz AN, Chou JF, et al. Phase II trial of hepatic artery infusional and systemic chemotherapy for patients with unresectable hepatic metastases from colorectal cancer: conversion to resection and long-term outcomes. Ann Surg 2015;261:353-360.

10. Kemeny NE, Niedzwiecki D, Hollis DR, Lenz HJ, Warren RS, Naughton MJ, et al. Hepatic arterial infusion versus systemic therapy for hepatic metastases from colorectal cancer: a randomized trial of efficacy, quality of life, and molecular markers (CALGB 9481). J Clin Oncol 2006;24:1395-1403.

11. Clavien PA, Selzner N, Morse M, Selzner M, Paulson E. Downstaging of hepatocellular carcinoma and liver metastases from colorectal cancer by selective intra-arterial chemotherapy. Surgery 2002;131:433-442.

12. Fernandes ESM, Line PD, Mello FP, Andrade RO, Girão CL, Pimentel LS, et al. Living donor liver transplant for colorectal liver metastasis: the first case in Latin America. Arq Bras Cir Dig 2019;32:e1468. 